\title{
ON THE STRUCTURE OF COMPLEX SOLVMANIFOLDS
}

\author{
KARL OELJEKLAUS \& WOLFGANG RICHTHOFER
}

\section{Introduction}

A connected complex space $X$ is called a solvmanifold if there is a connected solvable complex Lie group $G$ which acts holomorphically and transitively on it. The goal of this paper is to understand two special classes of solvmanifolds $X$ :

(1) $X$ is Kähler.

(2) $X$ is locally separable by global hypersurfaces, i.e., given $x \in X$ there are complex analytic hypersurfaces $F_{1}, \cdots, F_{k}$, smooth in $x$, such that $\bigcap_{i=1}^{k} F_{i}$ contains $x$ as an isolated point.

Our main result is the following

Main Theorem. Let $X$ be a complex solvmanifold which satisfies (1) or (2) and let $X \stackrel{F}{\rightarrow} Y$ denote the holomorphic reduction of $X$. Then $Y$ is a Stein manifold and $F$ is a Cousin group. Moreover the first fundamental group $\pi_{1}(X)$ contains a nilpotent subgroup of finite index.

A complex Lie group $G$ is called a Cousin group if $O(G)=$ C. In this case $G$ is abelian and one has $G=\mathrm{C}^{n} / \Gamma$, where $\Gamma$ is a certain discrete subgroup of $\mathbf{C}^{n}$ (see $\S 1$ ). The theorem is well known in the case where $X$ is compact (see [23], [7], [4]).

For $X$ noncompact the theorem was partially known when $X$ is a nilmanifold, i.e. when it admits a transitive holomorphic action of a nilpotent complex Lie group. A result of [8] states that for any nilmanifold $X$ the base of the holomorphic reduction $X \stackrel{F}{\longrightarrow} Y$ is Stein and the fiber $f$ has only constant holomorphic functions. Moreover $F$ is a principal abelian Lie group tower. In [17] it is shown that $F$ is already a Cousin group tower.

Using [8] and investigating the case of a nilmanifold $X$ with $O(X)=\mathbf{C}$, part (2) of the theorem was independently proved in [1] and [18] for the nilpotent case.

If $G$ is a nilpotent complex Lie group then it is easy to reduce function theoretic considerations to the case of discrete isotropy, i.e., $X=G / \Gamma$, where $\Gamma$ is a discrete subgroup of $G$. In this case there is a uniquely determined

Received January 6, 1986. 
connected real subgroup $G_{0}$ of $G$ containing $\Gamma$ cocompactly. This is not true in general for a solvable $G / \Gamma$.

The case where $G_{0}$ exists and is a real form of $G$ was considered in [13]. It is shown that $G / \Gamma$ satisfies (1) if and only if it is Stein. For $G / \Gamma$ satisfying (1) and $G_{0} / \Gamma$ a real hypersurface in $G / \Gamma$ the theorem was proved in [19].

The most recent result on solvmanifolds so far is given in [12]. It states that for any solvmanifold $X$ the base $Y$ of the holomorphic reduction $X \stackrel{F}{\rightarrow} Y$ is Stein. Moreover $\pi_{1}(Y)$ contains a nilpotent subgroup of finite index. In particular the theorem is true when $\operatorname{rank} O(X)=\operatorname{dim}_{\mathbf{C}} X$ (this condition already implies (1) and (2)).

The theorem yields striking results when the assumptions are more special. We give two corollaries as examples.

Corollary 1 (Conjecture of Ahiezer, see $\S 1$ ). Let $X$ be a solvmanifold satisfying (2) and suppose $O(X)=C$. Then $X$ is a Cousin group.

Corollary 2. Let $X=G / \Gamma$ be a solvmanifold. Suppose there exists a real form $G_{0}$ of $G$ containing $\Gamma$ cocompactly. Then $x$ satisfies (2) if and only if it is Stein.

To illustrate Corollary 2 we give the following example which is due to J. J. Loeb. For $K=\mathbf{Z}, \mathbf{R}, \mathbf{C}$ let $G_{K}=K \ltimes K^{2}$ be defined as follows: For $z, z^{\prime} \in K$ and $b, b^{\prime} \in K^{2}$ let

$$
(z, b) \cdot\left(z^{\prime}, b^{\prime}\right)=\left(z+z^{\prime}, e^{A z^{\prime}} b+b^{\prime}\right)
$$

where $A=\log \left(\begin{array}{ll}2 & 1 \\ 1 & 1\end{array}\right)$.

Then $G_{0}:=G_{\mathbf{R}}$ is a real form of $G=G_{\mathbf{C}}$ which contains $\Gamma=G_{\mathbf{Z}}$ cocompactly. Let $X=G / \Gamma$ and let $X \stackrel{F}{\rightarrow} Y$ denote the holomorphic reduction of $X$. J. J. Loeb proved that $Y=G / G^{\prime} \cdot \Gamma \cong \mathbf{C}^{*}$ and $F=G^{\prime} \cdot \Gamma / \Gamma \cong \mathbf{C}^{*} \times \mathbf{C}^{*}$. As far as we know, this is the first homogeneous counterexample to the Serre question whether a holomorphic fiber bundle with Stein base and Stein fiber is Stein. By Corollary 2 it follows that the hypersurface reduction of $X$ (see $\S 1$ ) has positive dimensional fibers. Since the fibration $G / \Gamma \rightarrow G / G^{\prime} \Gamma$ is the only possible for $G / \Gamma$ (see [21]), the holomorphic and the hypersurface reduction of $X$ coincide!

The method we use to prove our theorem is a combination of the methods in [11], [12], [13], [18] and [19].

We would like to thank D. Barlet who suggested we investigate the theory of currents in case (2) and A. T. Huckleberry who motivated us to find the final version of the Main Theorem. 


\section{Preliminaries}

Throughout this paper we are concerned with complex solvmanifolds, i.e. with homogeneous spaces $X=G / H$, where $G$ is a complex, solvable Lie group and $H$ a closed complex subgroup of $G$.

In this section we gather some basic definitions and known results about the complex analytic structure of $X=G / H$.

An important differential geometric concept for our approach is given in

Definition 1. A complex manifold $X$ is weakly Kähler if there is a closed, real, $(1,1)$-form $\omega$, which is positive semidefinite on $X$ and positive definite in at least one point $x \in X$.

Note that by continuity a form given as in Definition 1 is positive definite on some open set $U \subset X$.

It turns out that this condition is enough to obtain the conclusion of our Main Theorem for a complex solvmanifold $G / H$.

A basic technique to approach the structure of the algebra of holomorphic functions $O(X)$ of $X=G / H$ is the holomorphic reduction $G / H \stackrel{\pi}{\longrightarrow} G / J$ which is given by the following equivalence relation: two points $x, y \in X$ are equivalent if and only if for every $f \in O(X)$ one has $f(x)=f(y)$. This equivalence relation is Auto $(X)$-equivariant and the stabilizer $J \subset G$ of the equivalence-class of $x=H \in G / H$ is a closed complex subgroup of $G$ (see [8, Theorem 1.1]). The base of the holomorphic reduction is holomorphically separable and $O(G / H)=\pi^{*} O(G / J)$. Moreover $G / J$ is characterized by the universal property that every holomorphic map $F$ from $G / H$ into a holomorphically separable complex space factorizes through $G / J$. In general it is not known which reasonable conditions force the fiber $J / H$ of the holomorphic reduction to have only constant holomorphic functions.

Definition 2. A complex abelian Lie group $G$ with $O(G) \cong \mathbf{C}$ is called a Cousin group.

It is clear that a Cousin group $G$ is abelian and of the form $\mathbf{C}^{n} / \Gamma$, where $\Gamma$ is a discrete additive subgroup of $\mathbf{C}^{n}$. P. Cousin [5] was the first who considered such groups.

The following theorem is due to B. Gilligan and A. T. Huckleberry [8].

Theorem 3. Let $G$ be a nilpotent complex Lie group and $H$ a closed complex subgroup. Then there is a closed complex subgroup $J \subset G$ containing $H$, such that

(1) If $\pi: G / H \rightarrow G / J$ denotes the bundle projection, then $O(G / H)=$ $\pi^{*} O(G / J)$.

(2) $G / J$ is Stein.

(3) The fiber $J / H$ is connected. 
(4) $O(J / H) \cong \mathbf{C}$.

(5) $J / H$ is a principal abelian Lie group tower.

Remark. (a) In [18] it was shown that the fiber $J / H$ in Theorem 3 is a principal Cousin group tower.

(b) Recalling the example following Corollary 0.2 (Corollary 2 in §0) it is clear that this theorem is false in the solvable case, since the fiber of the holomorphic reduction may be a Stein manifold.

We give some facts which are known in the general solvable case. If $G$ is a (not necessarily closed) connected complex subgroup of $\mathrm{GL}_{n+1}(\mathbf{C})$, then every orbit of $G$ in $\mathbf{P}_{n}(\mathbf{C})$ is isomorphic to a product $\left(\mathbf{C}^{*}\right)^{k} \times \mathbf{C}^{l}$ [22]. Note that given $X=G / H$ and $N=N_{G}\left(H^{0}\right)$ is the normalizer of the identity component $H^{0}$ of $H$, then $G / N$ is equivariantly embedded into some $\mathbf{P}_{n}(\mathbf{C})$. We refer to $G / H \rightarrow G / N$ as the Tits fibration of $G / H$ (see [11]). From the remark above and the universality of the holomorphic reduction $G / H \rightarrow G / J$ it follows that $J \subset N$.

Proposition 4. Let $G$ be a simply connected solvable Lie group and $H$ a connected complex subgroup. Then $G / H \cong \mathbf{C}^{n}$.

Proof. This follows easily by Grauert's Oka principle and induction on $\operatorname{dim}_{\mathbf{C}} G / H$ using $[11$, I.2.5.8]. (Grauert's Oka principle states that a holomorphic fiber bundle over a Stein manifold having a complex structure group is holomorphically trivial if and only if it is topologically trivial [6].)

The most recent result about solvmanifolds $G / H$ gives in particular a characterization of the base $G / J$ of the holomorphic reduction, which satisfies the maximal rank condition. By definition, a complex $n$-dimensional manifold $X$ satisfies the maximal rank condition if there are $f_{1}, \cdots, f_{n} \in O(X)$ such that $d f_{1} \wedge \cdots \wedge d f_{n} \not \equiv 0$.

Theorem 5. Let $X=G / H$ be a complex solvmanifold satisfying the maximal rank condition. Then $X$ is Stein. Moreover $\pi_{1}(X)$ contains a nilpotent subgroup of finite index (see [12]).

To analyze the structure of the set $\mathcal{H}(X)$ of hypersurfaces in $X=G / H$ it is convenient to have a reduction analogous to the holomorphic reduction. For this we define the following equivalence relation: Two points $x, y \in X$ are equivalent if and only if for every $H \in \mathcal{H}(X)$ with $x \in H$ it follows $y \in H$ and vice versa. This equivalence relation yields a holomorphic equivariant fibration

$$
p: X=G / H \rightarrow G / I=Y
$$

such that $\mathcal{H}(X)=\pi^{*} \mathcal{H}(Y)$. Moreover $Y$ is separable by hypersurfaces, i.e. given $x, y \in Y, x \neq y$, there exists an $H \in H(Y)$ such that $x \in H, y \notin H$. (For details see [18, Kap. I].) A manifold $Y$ is said to be locally separable 
by hypersurfaces if for every point $y \in Y$ there are hypersurfaces $F_{1}, \cdots, F_{k}$ such that with $y \in F_{i}, i=1, \cdots, k$ and $\bigcap_{i=1}^{k} F_{i}$ contains $y$ as an isolated point. For a homogeneous manifold $X=G / H$ this condition implies that $X$ is weakly Kähler if $H^{0} \triangleleft G$. As in the case of holomorphic functions it is reasonable to ask for a description of $H(X)$ in terms of $G$ and $H$. The first result in this direction is due to Grauert and Remmert [18].

Theorem 6. Let $X$ be a compact complex homogeneous manifold. Then the hypersurface reduction $p: X \rightarrow Y$ is a holomorphic fiber bundle over a projective algebraic manifold $Y$ with connected complex parallelizable fiber.

Note that a homogeneous projective algebraic manifold $Y=G / I$ is a product $G / P \times T$, where $G / P$ is projective rational and $T$ is an abelian variety. The fibration $G / I \rightarrow G / P$ is just the Tits fibration of $Y$ and $P$ is the minimal parabolic subgroup of $G$ containing $I$ [4]. This result motivates the following general conjecture which is due to Ahiezer (see [1]):

Conjecture. Let $G$ be a complex Lie group and $H \subset G$ an arbitrary subgroup such that

(1) $O(G)^{H} \cong \mathbf{C}$, i.e., every right $H$-invariant holomorphic function on $G$ is constant.

(2) $H$ is not contained in a proper parabolic subgroup of $G$.

Then $\mathcal{H}(G)^{H}=\mathcal{H}(G)^{G^{\prime} \cdot H}$, i.e., every hypersurface $F \in \mathcal{H}(G)$ invariant under the right action of $H(F \cdot h=F, h \in H)$ is invariant under the right action of the commutator group $G^{\prime}$ of $G$.

If $G$ is solvable then condition (2) is redundant, since a complex solvable Lie group does not contain a proper parabolic subgroup.

This conjecture has been proved in several special cases. The case where $H$ is complex and cocompact in $G$ was described above. In this case it follows $P=G$ and $Y$ is an algebraic torus, i.e. $G^{\prime} \subset I$. If $G$ is semisimple, then $H$ is Zariski dense and the conjecture follows from the theorem of Huckleberry and Margulis [10].

The nilpotent case was handled in [1] and [18]. One of the main results of this paper is the proof of the conjecture for solvable $G$.

\section{CR-solvmanifolds}

To understand complex solvmanifolds, it is reasonable to study first those which inherit an additional structure and then to reduce the general situation to this case. In this section we study solvmanifolds $G / \Gamma$, which have a discrete isotropy and contain a compact generic $C R$-orbit of a subgroup $G_{0}$. To be precise we consider triples $\left(\Gamma, G_{0}, G\right)$ consisting of a simply connected, solvable, complex Lie group $G$, a (not necessarily complex) generic Lie group $G_{0}$ of $G$ 
and a discrete subgroup $\Gamma \subset G_{0}$, which is cocompact in $G_{0}$. Here a Lie subgroup $G_{0} \subset G$ is called generic if the Lie algebra $\mathfrak{g}_{0}$ of $G_{0}$ generates the Lie algebra $\mathfrak{g}$ as a vector space over $\mathbf{C}$, i.e.,

$$
\mathfrak{g}=\mathfrak{g}_{0}+i \mathfrak{g}_{0}
$$

The intersection $m:=\mathfrak{g}_{0} \cap i \mathfrak{g}_{0}$ is a complex ideal in $\mathfrak{g}$. A triple $\left(\Gamma, G_{0}, G\right)$ as above is called a $C R$-solvmanifold $(C R S)$.

If $\left(\Gamma, G_{0}, G\right)$ is a $C R S$ and $G / \Gamma$ is weakly Kählerian, we call $\left(\Gamma, G_{0}, G\right)$ a weakly Kählerian $C R$-solvmanifold (KCRS). Note that we may assume that the open set, where a given closed real positive semidefinite $(1,1)$ form $\omega$ is positive definite, intersects the compact $G_{0}$ orbit $G_{0} / \Gamma$. Identify $\omega$ with its right- $\Gamma$ invariant pullback to $G$. Since $G_{0} / \Gamma$ is compact it carries a left $G_{0}$-invariant, finite, normalized measure $\mu$.

Define $\tilde{\omega}$ on $G$ as

$$
\tilde{\omega}_{p}\left(v_{p}, w_{p}\right):=\int_{G_{0} / \Gamma} \omega_{p \cdot g}\left(d r_{g} v_{p}, d r_{g} w_{p}\right) d \mu(g),
$$

where $p \in G, v_{p}, w_{p} \in T_{p} G$ and the right- $\Gamma$ invariant function $G_{0} \rightarrow \mathbf{R}, g \mapsto$ $\omega_{p \cdot g}\left(d r_{g} v_{p}, d r_{g} w_{p}\right)$ is considered as a function on $G_{0} / \Gamma$. Let $\pi: G \rightarrow G / G_{0}$ denote the quotient map given by $G_{0}$. Furthermore let $V=\pi(U)$, where $U$ is an open right- $\Gamma$ invariant subset of $G$ such that $\omega \mid U$ is positive definite and $U \cap G_{0} \neq \varnothing$. Since $\pi$ is an open map $V$ is open in $G / G_{0}$ and $\tilde{U}=\pi^{-1}(U)$ is open and right- $G_{0}$ invariant in $G$. Let $p \in \tilde{U}$ and let $J$ denote the complex structure tensor on $G$. Then for $v \in T_{p} G$ we have

$$
\tilde{\omega}\left(v_{p}, J v_{p}\right)=\int_{G_{0} / \Gamma} \omega_{p \cdot g}\left(d r_{g} v_{p}, J d r_{g} v_{p}\right) d \mu(g)>0,
$$

since $\omega \mid \tilde{U} \geq 0$ and $\tilde{U} \cap U \neq \varnothing$. Moreover, $\tilde{\omega}$ is a closed real positive semidefinite $(1,1)$ form which is right- $G_{0}$ invariant. These considerations show that $\left(\Gamma, G_{0}, G\right)$ is $K C R S$ if and only if there is a closed real positive semidefinite right- $G_{0}$ invariant $(1,1)$ form $\omega$ on $G$ and a $G_{0}$-right invariant open neighborhood of $G_{0}$ in $G$, where $\omega$ is positive definite.

In the following we always assume a $K C R S$ to be given in such a way.

For the statement of the main result of this section it is necessary to introduce some further notation.

Definition 1. A real Lie algebra $\mathfrak{g}$ has an imaginary spectrum if every $v \in \operatorname{ad}(\mathfrak{g})$ considered as an endomorphism of $\mathfrak{g}$ has only imaginary eigenvalues (note that zero is also an imaginary number).

The main result of this section is

Theorem 2. Let $\left(\Gamma, G_{0}, G\right)$ be a KCRS. Then $\mathfrak{g}_{0}$ has an imaginary spectrum. 
It is essential for the proof of Theorem 2 to characterize those real Lie algebras which do not have an imaginary spectrum and to describe the function theoretic properties of those CRS's in which they appear. At first we give two examples of such Lie algebras:

Examples 3. (i) Let $A_{\mathbf{R}}$ denote the group of affine transformations of the real line. Then clearly the Lie algebra $\mathfrak{a}$ of $A_{\mathbf{R}}$ does not have an imaginary spectrum.

(ii) For $\mu \in \mathbf{R} \backslash\{0\}$ let $G_{\mu}$ denote the Lie group defined as follows. Take $\mathbf{R} \times \mathbf{R}^{2}$ and define a multiplication on it by

$$
(z, b)\left(z^{\prime}, b^{\prime}\right)=\left(z+z^{\prime}, e^{z^{\prime} A_{\mu}} \cdot b+b^{\prime}\right)
$$

where $z, z^{\prime} \in \mathbf{R}, b, b^{\prime} \in \mathbf{R}^{2}$ and $A_{\mu}:=\left(\begin{array}{cc}1 & \mu \\ -\mu & 1\end{array}\right)$. Then the Lie algebra $\mathfrak{g}_{\mu}$ of $G_{\mu}$ does not have an imaginary spectrum. q.e.d.

The following theorems are due to J. J. Loeb (see [13]).

Theorem 4. Let $G$ be a simply connected real Lie group. Then the Lie algebra $\mathfrak{g}$ of $G$ does not admit an imaginary spectrum if and only if $G$ contains a subgroup of the form $A_{\mathbf{R}}$ or $G_{\mu}$.

Theorem 5. Let $A_{\mathbf{C}}$ (resp. $G_{\mu}^{\mathbf{C}}$ ) denote the complexification of $A_{\mathbf{R}}$ (resp. $\left.G_{\mu}\right)$ and let $U$ denote a right- $A_{\mathbf{R}}$ (resp. $\left.G_{\mu}\right)$ invariant open neighborhood of $A_{\mathbf{R}}\left(\right.$ resp. $\left.G_{\mu}\right)$ in $A_{\mathbf{C}}\left(\right.$ resp. $\left.G_{\mu}^{\mathbf{C}}\right)$. Then there does not exist any positive closed $(1,1)$ form $\omega$ on $A_{\mathbf{C}}\left(\right.$ resp. $\left.G_{\mu}^{\mathbf{C}}\right)$ which is right- $A_{\mathbf{R}}\left(\right.$ resp. $\left.G_{\mu}\right)$ invariant and strictly positive on $U$.

This statement is not explicit in [13], but it follows from [13] by proof of Theorem 5, Proposition 3 together with its corollary (in the case $A_{\mathbf{R}}, A_{\mathbf{C}}$ ) and Proposition 4 together with the Remark (case $G_{\mu}, G_{\mu}^{\mathbf{C}}$ ).

The last ingredient we need for the proof of Theorem 2 is

Proposition 6. Let $G$ be a complex Lie group. Suppose that $G$ admits a Kähler metric which is invariant with respect to the right (or left) multiplication of $G$. Then $G$ is abelian.

Proof. Let $h$ denote such a Kähler metric on $G$. Assume that it is right- $G$ invariant. Consider the right invariant vector fields on $G$ and identify them with the Lie algebra $\mathfrak{g}$ of $G$. The Kähler form $\omega$ associated to $h$ is given by $\omega(X, Y)=h(X, J Y)$ where $J$ denotes the complex structure tensor on $G$. Since $\omega$ is closed and right invariant we have

$$
0=\omega(X,[Y, Z])+\omega(Z,[X, Y])+\omega(Y,[Z, X])
$$

for all $X, Y, Z \in \mathfrak{g}$. 
Assume there is a nonabelian, two-dimensional, complex subalgebra $\mathfrak{a} \subset \mathfrak{g}$. Then there is a basis $(X, Y)$ of $\mathfrak{a}$ satisfying $[X, Y]=Y$. By $(*)$ we have

$$
\begin{aligned}
0 & =\omega(X,[Y, J Y])+\omega(J Y,[X, Y])+\omega(Y,[J Y, X]) \\
& =2 \omega(J Y, Y)=2 h(J Y, J Y)>0,
\end{aligned}
$$

which is a contradiction. Thus every two-dimensional complex subalgebra of $\mathfrak{g}$ is abelian. Let $B$ denote a maximal solvable subalgebra of $\mathfrak{g}$. We claim that $B$ is nilpotent. To see this assume the contrary. Then there is a $k \in \mathbf{N}$ such that $\left[B, B^{(k)}\right]=B^{(k)}$, where $B^{(k)}$ denotes the descending central series of $B$. Lie's flag theorem applied to the adjoint representation of $B$ in $\operatorname{End}\left(B^{(k)}\right)$ yields the existence of a nonabelian two-dimensional complex subalgebra of $B$. This contradiction proves our claim, and $B$ being nilpotent implies that $\mathfrak{g}$ is nilpotent.

Now $\mathfrak{g}$ being nilpotent, it has a nontrivial center $C$, which nontrivially intersects every nonzero ideal of $\mathfrak{g}$, in particular the commutator algebra $\mathfrak{g}^{\prime}$ if it is nontrivial. Let $0 \neq X \in C \cap \mathfrak{g}^{\prime}$. Then by $(*)$ we have

$$
0=\omega(X,[Y, Z])
$$

for all $Y, Z \in \mathfrak{g}$. Thus $\omega\left(X, X^{\prime}\right)=0$ for all $X^{\prime} \in \mathfrak{g}^{\prime}$. Since $\omega \mid \mathfrak{g}^{\prime}$ is nondegenerate, this shows that $\mathfrak{g}^{\prime}=0$ and $G$ is abelian.

Corollary 7. Let $\left(\Gamma, G_{0}, G\right)$ be a KCRS. Then $\mathfrak{g}_{0} \cap i \mathfrak{g}_{0}=m$ is abelian.

Proof. Let $G_{m}=\langle\exp m\rangle$ denote the Lie subgroup of $G_{0}$ generated by $m$. Take a $G_{0}$-right invariant neighborhood $U$ of $G_{0}$ in $G$ and a closed positive $G_{0}$-right invariant $(1,1)$ form $\omega$ which is strictly positive definite on $U$. Then $\omega \mid G_{m}$ is right $G_{m}$ invariant. Since $G_{m}$ is a complex Lie group, the corollary now follows from Proposition 6 . q.e.d.

We now come to the

Proof of Theorem 2. Assume that $\mathfrak{g}_{0}$ does not have an imaginary spectrum. Then $G_{0}$ contains a subgroup of the form $A_{\mathbf{R}}$ or $G_{\mu}$. At first assume it contains $A_{\mathbf{R}}$. Let $A$ denote the complexification of $A_{\mathbf{R}}$ in $G$, i.e., $A=$ $\langle\exp (\mathfrak{a}+i \mathfrak{a})\rangle$, where the sum is not necessarily direct. By a dimension argument and by Proposition 6 it follows that $A_{\mathbf{R}}$ is a real form of $A$ (otherwise $A_{\mathbf{R}}$ would be abelian). This however contradicts Theorem 5 .

Now take $G_{\mu} \subset G_{0}$ and its complexification $G_{\mu}^{\mathbf{C}}$ in $G$. Again by Theorem 5 $G_{\mu}$ cannot be a real form of $G_{\mu}^{\mathbf{C}}$. Thus $G_{\mu}^{\mathbf{C}}$ has to be a two-dimensional complex subgroup of $G$. Since a complex two-dimensional nonabelian Lie subalgebra contains exactly one nonzero ideal, namely its commutator algebra and since $\mathfrak{g}_{\mu} \cap i \mathfrak{g}_{\mu} \neq 0$ in this case, it follows that

$$
\left(\mathfrak{g}_{\mu}^{\mathbf{C}}\right)^{\prime}=\mathfrak{g}_{\mu} \cap i \mathfrak{g}_{\mu} .
$$


Also since $\mathfrak{g}_{\mu}^{\mathbf{C}}$ is two dimensional, every eigenvector in $\mathfrak{g}_{\mu}^{\mathbf{C}}$ belonging to a nonzero eigenvalue of an element ad $X, X \in \mathfrak{g}_{\mu}^{\mathbf{C}}$, has to be contained in $\left(\mathfrak{g}_{\mu}^{\mathbf{C}}\right)^{\prime}$. Take $X \in \mathfrak{g}_{\mu}$ and let $\lambda \in \mathbf{C}$ be a nonzero eigenvalue of ad $X$. Then there is a $Y \in\left(\mathfrak{g}_{\mu}^{\mathbf{C}}\right)^{\prime}$ such that $[X, Y]=\lambda \cdot Y$.

Let $\lambda=\alpha+i \beta, \alpha, \beta \in \mathbf{R}$. We have

$$
0=\omega(X,[Y, i Y])+\omega(i Y,[X, Y])+\omega(Y,[i Y, X])
$$

because $Y \in \mathfrak{g}_{\mu} \cap i \mathfrak{g}_{\mu} \subset \mathfrak{g}_{0}$.

Hence it follows that

$$
0=2 \omega(i Y,[X, Y])=2 \omega(i Y, \alpha Y+i \beta Y)=2 \alpha \omega(i Y, Y) .
$$

Since $\omega(i Y, Y)>0$ it follows that $\alpha=0$. Hence ad $X$ has only imaginary eigenvalues, which contradicts the fact that $\mathfrak{g}_{\mu}$ has a nonimaginary spectrum.

Therefore by Theorem 4 it follows that $\mathfrak{g}_{0}$ has an imaginary spectrum.

Remark 8. Theorem 2 was proved by Loeb in the case where $G_{0}$ is a real form of $G$. In this case one has the equivalence: $G / \Gamma$ is Kähler if and only if $\mathfrak{g}_{0}$ has an imaginary spectrum (see [13]).

For later use we need the following:

Lemma 9. Let $\left(\Gamma, G_{0}, G\right)$ be a $C R S$ and let $H$ be a closed complex subgroup of $G$ containing $\Gamma$. Let $H_{0}:=H \cap G_{0}$. Then $G_{0} / H_{0}$ is totally real in $G / H$ if and only if the following conditions are satisfied:

(i) $\mathfrak{h}_{0}+i \mathfrak{h}_{0}=\mathfrak{h}$.

(ii) $m \subset \mathfrak{h}$.

Proof. The $G_{0}$-orbit of $p=H$ in $G / H$ is totally real if and only if $0=$ $m_{p}:=T_{p}\left(G_{0} / H_{0}\right) \cap i T_{p}\left(G_{0} / H_{0}\right)$. Since $T_{p}\left(G_{0} / H_{0}\right)+i T_{p}\left(G_{0} / H_{0}\right)=T_{p}(G / H)$, we have

$$
\begin{aligned}
\operatorname{dim}_{\mathbf{R}} m_{p} & =2 \operatorname{dim}_{\mathbf{R}} T_{p}\left(G_{0} / H_{0}\right)-\operatorname{dim}_{\mathbf{R}} T_{p}(G / H) \\
& =\operatorname{dim}_{\mathbf{R}} \mathfrak{g}_{0}-2 \operatorname{dim}_{\mathbf{R}} \mathfrak{h}_{0}-\operatorname{dim}_{\mathbf{R}} \mathfrak{g}+\operatorname{dim}_{\mathbf{R}} \mathfrak{h} .
\end{aligned}
$$

On the other hand $\operatorname{dim}_{\mathbf{R}} m=2 \operatorname{dim}_{\mathbf{R}} \mathfrak{g}_{0}-\operatorname{dim}_{\mathbf{R}} \mathfrak{g}$, since $G_{0}$ is a generic subgroup of $G$. Thus we obtain

$$
\operatorname{dim}_{\mathbf{R}} m_{p}=\operatorname{dim}_{\mathbf{R}} m-2 \operatorname{dim}_{\mathbf{R}} \mathfrak{h}_{0}+\operatorname{dim}_{\mathbf{R}} \mathfrak{h}
$$

and $\operatorname{dim}_{\mathbf{R}} m_{p}=0$ if and only if

$$
0=\operatorname{dim}_{\mathbf{R}} m-2 \operatorname{dim}_{\mathbf{R}} \mathfrak{h}_{0}+\operatorname{dim}_{\mathbf{R}} \mathfrak{h} .
$$

Note that $\operatorname{dim}_{\mathbf{R}}\left(\mathfrak{h}_{0}+i \mathfrak{h}_{0}\right)=2 \operatorname{dim}_{\mathbf{R}} \mathfrak{h}_{0}-\operatorname{dim}_{\mathbf{R}}\left(\mathfrak{h} \cap i \mathfrak{h}_{0}\right)$, i.e. $m_{p}=0$ if and only if

$$
0=\operatorname{dim}_{\mathbf{R}} m-\operatorname{dim}_{\mathbf{R}}\left(\mathfrak{h}_{0}+i \mathfrak{h}_{0}\right)-\operatorname{dim}_{\mathbf{R}}\left(\mathfrak{h}_{0} \cap i \mathfrak{h}_{0}\right)+\operatorname{dim}_{\mathbf{R}} \mathfrak{h}
$$


Since $\mathfrak{h}_{0}+i \mathfrak{h}_{0} \subset \mathfrak{h}$ and $\mathfrak{h}_{0} \cap i \mathfrak{h}_{0} \subset m$ the last equation holds if and only if

(i) $\mathfrak{h}_{0}+i \mathfrak{h}_{0}=\mathfrak{h}$;

(ii) $\mathfrak{h}_{0} \cap i \mathfrak{h}_{0}=m$.

Remark 10. Let $\left(\Gamma, G_{0}, G\right)$ be a $C R S$ and $H, H_{0}$ be given as in Lemma 2.7. If moreover $\mathfrak{h}_{0}$ is an ideal in $\mathfrak{g}_{0}$, then $\left(H_{0} / H_{0}^{0}, G_{0} / H_{0}^{0}, G / H^{0}\right)$ is a $C R S$ and $G_{0} / H_{0}^{0}$ is a real form of $G / H^{0}$, where $H_{0}^{0}$ denotes the identity component of $H_{0}$.

\section{Nilmanifolds}

In this section we consider weakly Kählerian nilmanifolds. A nilmanifold $X$ is a quotient space $X=G / H$, where $G$ is a nilpotent complex Lie group and $H \subset G$ a closed complex subgroup. (Of course we may assume that $G$ is simply connected). Let $\pi: G / H \rightarrow G / J$ denote the holomorphic reduction of $G / H$. Then $O(J / H)=\mathrm{C}, G / J$ is Stein and $J / H$ is connected (see Theorem 1.3). If $G / H$ is weakly Kähler then clearly the same is true for $J / H$.

Our aim is to proof

Theorem 1. Let $X$ be a weakly Kählerian nilmanifold and let $X \stackrel{F}{\rightarrow} Y$ denote the holomorphic reduction of $X$. Then $Y$ is Stein and $F$ is a Cousin group.

Note that $O(J / H)=\mathbf{C}$ implies that $H^{0} \triangleleft J^{0}$. If $G$ is simply connected then the same is true for $J^{0} / H^{0}$ and we have $J / H=\tilde{G} / \tilde{\Gamma}$, where $\tilde{G}=J^{0} / H^{0}$ and $\tilde{\Gamma}=J^{0} \cap H$. Thus Theorem 1 immediately follows from

Theorem 2. Let $G$ be a simply connected complex nilpotent Lie group and $\Gamma \subset G$ a discrete subgroup such that

(1) $O(G / \Gamma)=\mathbf{C}$ and

(2) $G / \Gamma$ is weakly Kählerian.

Then $G$ is abelian.

Indeed the assumption in Theorem 2 describes a special $K C R S$. For this we quote Theorem 3 [15]: Let $G$ be a simply connected nilpotent Lie group and $H \subset G$ a closed subgroup. Then there exists a unique connected closed subgroup $G_{0} \subset G$ containing $H$, such that

(1) $G_{0} / H$ is compact;

(2) $G / G_{0} \cong \mathbf{R}^{n}$;

(3) The bundle $G / H \rightarrow G / G_{0}$ is real analytically trivial.

Note that having such a $G_{0}$ in the situation of Theorem 2 implies that $G_{0}^{\mathbf{C}}=\left\langle\exp g_{0}+i \mathfrak{g}_{0}\right\rangle$ is a closed complex subgroup of $G$ containing $\Gamma$. Thus $G / G_{0}^{\mathbf{C}}=\mathbf{C}^{k}(1.4)$ and the condition $O(G / \Gamma)=\mathbf{C}$ forces $G_{0}^{\mathbf{C}}=G$, i.e., $G_{0}$ 
is a generic subgroup of $G$. Thus we have to prove an equivalent version of Theorem 2.

Theorem 2'. Let $\left(\Gamma, G_{0}, G\right)$ be a KCRS. Suppose $G$ is nilpotent and $O(G / \Gamma)=$ C. Then $G$ is abelian.

Proof. Let $\omega$ denote a right- $G_{0}$ invariant closed real positive $(1,1)$ form on $G$, which is strictly positive on a right- $G_{0}$ invariant open neighborhood $U$ of $G_{0}$ in $G$. For $p \in G$ and $v_{p}, w_{p} \in T_{p} G$ define

$$
h\left(v_{p}, w_{p}\right)=\omega\left(J v_{p}, w_{p}\right),
$$

where here $J$ denotes the complex structure tensor on $G$. Then $h \mid U$ is a right- $G_{0}$ invariant Kähler metric on $U$.

Since $G_{0}$ is a generic subgroup of $G$, the center $C_{0}$ of $\mathfrak{g}_{0}$ is given by $C_{0}=$ $C \cap \mathfrak{g}_{0}$, where $C$ denotes the center of $\mathfrak{g}$.

At first we show that

$$
C \cap m=\{X \in m: \omega(X,[\mathfrak{g}, m])=0\} .
$$

For this let

$$
\mathfrak{a}=\left\{X \in \mathfrak{g}_{0}: \omega(X, m)=0\right\} .
$$

Then $\mathfrak{a}$ is a real subalgebra of $\mathfrak{g}_{0}$ and we have $\mathfrak{g}_{0}=\mathfrak{a} \times m$. To see this consider $h$ and let $\left(v_{1}, \cdots, v_{2 k}, w_{1}, \cdots, w_{l}\right)$ be an orthonormal basis of $\mathfrak{g}_{0}$, where $\left(v_{1}, \cdots, v_{2 k}\right)$ is an orthonormal basis of $m$, such that $v_{j+k}=J v_{j}$ for $j \leq k$.

Using the relation between $\omega$ and $h$, it is easy to see that $\mathfrak{a}$ is just the real span of $\left(w_{1}, \cdots, w_{l}\right)$. Thus $\mathfrak{g}_{0}=\mathfrak{a} \oplus m$ as a vector space. The fact that $\mathfrak{a}$ is an algebra follows from the formula

$$
0=\omega(X,[Y, Z])+\omega(Z,[X, Y])+\omega(Y,[Z, X])
$$

for all $X, Y, Z \in \mathfrak{g}_{0}$, using the fact that $m$ is an ideal in $\mathfrak{g}_{0}$. (Note that $[\mathfrak{g}, m]=\left[\mathfrak{g}_{0}, m\right]$. Thus, using Corollary 2.7 , which says that $m$ is abelian, we have $[\mathfrak{g}, m]=[\mathfrak{a}, m]$.

By $(* *)$ it follows that

$$
C \cap m \subset\{X \in m: \omega(X,[\mathfrak{g}, m])=0\} .
$$

To verify the opposite inclusion it is enough to show that for $X_{0} \in\{X \in$ $m: \omega(X, m)=0\}$ one has $\left[X_{0}, \mathfrak{a}\right]=0$. For this let $Y \in \mathfrak{a}$ and $Z \in m$. Then by $(* *)$ we have

$$
0=\omega\left(X_{0},[Y, Z]\right)+\omega\left(Z,\left[X_{0}, X\right]\right)+\omega\left(Y,\left[Z, X_{0}\right]\right) .
$$

Since $[Y, Z] \in[\mathfrak{g}, m]$ and $\left[Z, X_{0}\right] \in m$, it follows that

$$
\omega\left(Z,\left[X_{0}, Y\right]\right)=0
$$

for all $Z \in m, Y \in \mathfrak{a}$. 
But $X_{0} \in m$ implies that $\left[X_{0}, Y\right] \in m$. Since $m$ is a complex subspace of $\mathfrak{g}$, we know that $\omega \mid m$ is nondegenerate. Therefore we have $\left[X_{0}, Y\right]=0$ and $(*)$ is proved.

Since $\mathfrak{g}$ is nilpotent, we have $C \neq 0$ and $C$ nontrivially intersects every nonzero ideal of $\mathfrak{g}$. Thus $[\mathfrak{g}, m]$ being an ideal in $\mathfrak{g}$ implies $[\mathfrak{g}, m]=0$ (see $(*))$ or equivalently that $m \subset C$.

We now claim that

$$
C=C_{0}+i C_{0} .
$$

For this note that $\mathfrak{g}_{0}^{\prime}=\mathfrak{a}^{\prime}+[\mathfrak{a}, m]$. Since $[\mathfrak{a}, m]=0$, it follows that $\mathfrak{g}_{0}^{\prime} \cap i \mathfrak{g}_{0}^{\prime}=$ $\mathfrak{a}^{\prime} \cap i \mathfrak{a}^{\prime} \cap m \subset \mathfrak{a} \cap m=0$.

Take $C \ni Z=X+i Y, X, Y \in \mathfrak{g}_{0}$. Then for an arbitrary $X^{\prime} \in \mathfrak{g}_{0}$ we have

$$
0=\left[Z, X^{\prime}\right]=\left[X, X^{\prime}\right]+i\left[Y, X^{\prime}\right]
$$

which shows that $\left[X, X^{\prime}\right],\left[Y, Y^{\prime}\right] \in \mathfrak{g}_{0}^{\prime} \cap i \mathfrak{g}_{0}^{\prime}=0$. Thus $X, Y \in C_{0}$, which proves our claim.

Note that $O(G / \Gamma)=\mathbf{C}$ implies that the orbits of the center $Z$ of $G$ are closed in $G / \Gamma[2]$. Thus we may consider the fibrations:

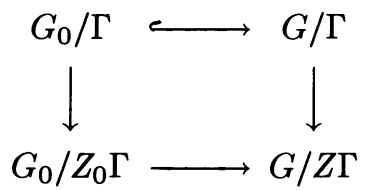

Since $m \subset C$, and $C_{0}+i C_{0}=C$, it follows from Lemma 2.9 that $G_{0} / Z_{0}$ is a real form of $G / Z$. Thus $G / Z \Gamma$ is Stein $[8$, p. 47] and $O(G / \Gamma)=\mathbf{C}$ forces $G=Z$, i.e., $G$ is abelian.

Remarks 4. (a) ${ }^{1}$ It should be noted that the proof of Theorem $2^{\prime}$ yields an exact description of Kählerian nilmanifolds.

Let $G / H$ be a complex nilmanifold. Then there exists a uniquely determined nilpotent complex Lie group $N$, such that $G / H=\mathrm{C}^{n} \times N / \Gamma$, where $\Gamma$ is a "maximal" discrete subgroup of $N$. (Here "maximal" means that $\Gamma$ is not contained in a proper connected complex subgroup of $N$ ) (see [14]). Thus $G / H$ is Kähler if and only if $N / \Gamma$ is Kähler. Now let $N_{0}$ denote the smallest connected real subgroup of $N$ containing $\Gamma$ (see Theorem 3). By the proof of Theorem $2^{\prime}$ it follows that the Kähler assumption on $G / H$ yields a splitting

$$
n_{0}=\mathfrak{a} \oplus m, \quad \text { where } m \text { is abelian and } \mathfrak{a} \cap i \mathfrak{a}=0 .
$$

Conversely assume that there is such a splitting of $n_{0}$. Let $\mathfrak{a}^{\mathbf{C}}=\mathfrak{a}+i \mathfrak{a}$ and $A$ $\left(\operatorname{resp} . A^{\mathbf{C}}\right)=\langle\exp \mathfrak{a}\rangle\left(\operatorname{resp} .\left\langle\exp \mathfrak{a}^{\mathbf{C}}\right\rangle\right)$. Then we have

$$
N_{0}=A \times G_{m} \text { and } \quad N=A^{\mathbf{C}} \times G_{m} .
$$

\footnotetext{
${ }^{1}$ We thank J. J. Loeb for contributing to remark (a).
} 
Moreover there exists a right $A$-invariant Kähler form on $A^{\mathbf{C}}$ (see [13]). Since $G_{m}$ is abelian this yields a right $N_{0}$-invariant Kähler form on $N$ and hence a Kähler form on $N / \Gamma$.

(b) For practical use it is convenient to note that the Kähler assumption on $N / \Gamma$ implies that $n_{0}^{\prime} \cap m=0$.

(c) Remark (a) shows that a nilmanifold is weakly Kählerian if and only if it is Kählerian.

(d) The formula $(*)$ in the proof of Theorem $2^{\prime}$ is valid for every $K C R S$ $\left(\Gamma, G_{0}, G\right)$. One can say a little bit more about the splitting $\mathfrak{g}_{0}=\mathfrak{a} \ltimes m$ in the case where $G$ is not nilpotent.

Let $n$ (resp $n_{\mathfrak{a}}$ ) denote the nilradical of $\mathfrak{g}_{0}$ (resp. a). Then $n=n_{\mathfrak{a}} \ltimes m$. The arguments in the proof of Theorem 2 show that $n=n_{\mathfrak{a}} \oplus m$. Hence the adjoined action of $\mathfrak{g}_{0}$ on $m$ factorizes through $\mathfrak{g}_{0} / n=\mathfrak{a} / n_{\mathfrak{a}}$. It is easy to see that the eigenvalues of an element $a \in \mathfrak{a}$ are purely imaginary and (*) implies that $\mathfrak{g}_{0}=\mathfrak{a} \oplus m$ if and only if there is some $X \in m$, such that $[a, X]=0$.

\section{The holomorphic reduction of Kählerian solvmanifolds}

In this section we prove

Theorem 1. Let $G / H$ be a weakly Kählerian solvmanifold. Then the holomorphic reduction $G / H \rightarrow G / J$ realizes $G / H$ as a Cousin group bundle over a Stein manifold. Moreover $\pi_{1}(G / H)$ contains a subgroup of finite index which is nilpotent.

The proof of Theorem 1 uses results and methods of [12], which essentially reduce the general situation to that of a $K C R S$. At first we recall some basic notions, which are essential for this reduction.

An element $g$ of a complex Lie group $G$ is called regular if the Zariski closure of $\mathbf{Z}_{g}:=\left\{\operatorname{Ad} g^{n}: n \in \mathbf{Z}\right\}$ in $\mathrm{GL}_{\mathbf{C}}(\mathfrak{g})$ contains a maximal torus of the Zariski closure of $\operatorname{Ad}(G)$. (For details about this notion we refer to [17].)

Let $G$ be a simply connected solvable complex Lie group. Then there exists a solvable linear algebraic complex Lie group

$$
G_{a}=\left(\mathbf{C}^{*}\right)^{k} \ltimes G
$$

containing $G$ as a Zariski dense and topologically closed normal subgroup (see [9, Theorem 3.1]). The group $G_{a}$ is called a regular algebraic hull of $G$. The commutator subgroups of $G_{a}$ and $G$ coincide (see [3]). For every closed complex subgroup $H \subset G$ the complex manifolds $G_{a} / H$ and $\left(\mathbf{C}^{*}\right)^{k} \times G / H$ are biholomorphically equivalent. In particular $G_{a} / H$ is weakly Kähler if and only if $G / H$ is weakly Kähler. If $G_{a} / H \rightarrow G_{a} / J$ denotes the holomorphic 
reduction, then $J \subset G$ and $G_{a} / J=\left(\mathbf{C}^{*}\right)^{k} \times G / J$. It is also clear that $\pi_{1}(G / H)$ is nilpotent if and only if $\pi_{1}\left(G_{1} / H\right)$ is nilpotent.

A basic step for the proof of Theorem 1 is

Lemma 2. Let $G / \Gamma$ be a weakly Kählerian solvmanifold. Assume $G$ is simply connected and $\Gamma$ is a discrete subgroup of $G$, which contains a regular element. Then $\Gamma$ contains a nilpotent subgroup $\tilde{\Gamma}$ of finite index.

This lemma is essentially due to A. Huckleberry and E. Oeljeklaus. In [12] it is proved for the special case, where $\operatorname{rank} O(G / \Gamma)=\operatorname{dim}_{\mathbf{C}} G / \Gamma$. However the proof basicly uses the fact that for a $C R S\left(\Gamma, G_{0}, G\right)$, where $G / \Gamma$ satisfies the maximal rank condition, the Lie algebra $\mathfrak{g}_{0}$ has purely imaginary spectrum [13]. Since more general $\left(\Gamma, G_{0}, G\right)$ being a $K C R S$ implies that $g_{0}$ has an imaginary spectrum (see Theorem 2.2), the proof of [12] carries over to the situation of Lemma 2.

Lemma 3. Let $G / \Gamma$ be a weakly Kählerian solvmanifold. Suppose $G$ is an algebraic group and $\Gamma$ is a discrete Zariski dense subgroup of $G$. Then $G$ is nilpotent.

This lemma is also proved in [12] for the case where $\operatorname{rank} O(G / \Gamma)=$ $\operatorname{dim}_{\mathbf{C}} G / \Gamma$. However the proof only uses Lemma 2 and the fact that a Zariski dense subgroup of a connected complex algebraic group contains a regular element.

We now come to the

Proof of Theorem 1. At first we may assume that $G$ is simply connected. Note also that taking a complex subgroup $\tilde{H} \subset H$ of finite index instead of $H$ does not have any effect on the statement of the theorem. Let $G_{a}=\left(\mathbf{C}^{*}\right)^{k} \ltimes G$ denote a regular algebraic hull of $G$. For any subgroup $L \subset G$, let $G_{a}(L)$ denote the Zariski closure of $L$ in $G_{a}$. The group $H_{0}:=\left(G_{a}(H)\right)^{0} \cap H$ has a finite index in $H$. Thus by the remark above we may assume that $H_{0}=H$ and $G_{a}(H)$ is connected.

Let us now consider the case where $H$ is a discrete subgroup of $G$. It is clear that $G_{a}(H) / H$ is weakly Kähler. Thus by Lemma 3 it follows that $G_{a}(H)$ is nilpotent. Let $G_{a}(H) / H \rightarrow G_{a}(H) / J$ denote the holomorphic reduction of the $G_{a}(H)$ orbit of $H$ in $G_{a} / H$. Then by Theorem 3.1 we know that $G_{a}(H) / J$ is Stein and $J / H$ is a Cousin group. Since quotients of solvable, algebraic groups are Stein and $G_{a}(H)$ is an algebraic subgroup of $G_{a}$ we also know that $G_{a} / G_{a}(H)$ is Stein (see [11], [22]). Thus we have the holomorphic fibrations

$$
G_{a} / H \rightarrow G_{a} / J \rightarrow G_{a} / G_{a}(H) .
$$

A basic theorem of Matsushima and Morimoto [16] says the following: Let $E \rightarrow B$ be a holomorphic fiber bundle with fiber $F$ and a complex Lie group $S$ 
a structure group. Assume that $S / S^{0}$ is finite and $F$ and $B$ are Stein. Then $E$ is Stein.

The application of this theorem to the bundle $G_{a} / J \rightarrow G_{a} / G_{a}(H)$ shows that $G_{a} / J$ is Stein. Thus $G_{a} / H \rightarrow G_{a} / J$ is the holomorphic reduction. By the remark previous to Lemma 2 we have that $J \subset G$, thus $G / J$ is Stein, $J / H$ is a Cousin group and $H$ is nilpotent $\left(H \subset G_{a}(H)\right.$ !).

If $H$ is not discrete in $G$ let $N=N_{G_{a}}\left(H^{0}\right)$. This is an algebraic subgroup of $G_{a}$ (see [12]) and $H / H^{0}$ is a discrete subgroup of $N / H^{0}$. Since also $N^{0}$ is algebraic and $H \subset G_{a}(H)$ which is already assumed to be connected, it follows that $H \subset N^{0}$. Thus, by the above, we know that the holomorphic reduction $N^{0} / H \rightarrow N^{0} / J$ has a Stein base and a Cousin group as fiber. Since $G_{a} / N^{0}$ is Stein it follows (again by the theorem of Matsushima-Morimoto) that $G_{a} / J$ is Stein and $G_{a} / H \rightarrow G_{a} / J$ is the holomorphic reduction. Thus again $J \subset G, G / J$ is Stein and $G / H \rightarrow G / J$ is the holomorphic reduction of $G / H$. Moreover, by the above we have that $H / H^{0}$ contains a nilpotent subgroup $H$ of finite index. Thus $\pi_{1}(G / H) \cong H / H^{0}$ (note that $G \cong_{\text {bihol }} \mathbf{C}^{n}$ ) contains a nilpotent subgroup of finite index and the theorem is proved.

Corollary 4 [12]. Let $G$ be a solvable complex Lie group and $H \subset G$ a closed complex subgroup. Then $G / H$ satisfies the maximal rank condition if and only if it is Stein. In this case $\pi_{1}(G / H)$ admits a nilpotent subgroup of finite index.

Corollary 5. Let $G$ be a solvable complex Lie group and $H \subset G$ a closed complex subgroup such that $G / H$ is weakly Kähler and $O(G / H)=\mathrm{C}$. Then $G^{\prime} \subset H$ and $G / H$ is a Cousin group.

Finishing this section we give two examples of nilmanifolds realized as Cousin group bundles over Stein manifolds. The first is Kähler and the second is not. These examples show that the converse of Theorem 1 is not true in general and furthermore that the bundle given by Theorem 1 may be not holomorphically trivial.

Examples 6. (a) Consider the group of matrices

$$
G_{K}:=\left\{\left(\begin{array}{cccc}
1 & x & z & w \\
0 & 1 & y & 0 \\
0 & 0 & 1 & 0 \\
0 & 0 & 0 & 1
\end{array}\right): x, y, z, w \in K\right\}, \quad \text { where } K=\mathbf{C}, \mathbf{R}, \mathbf{Z}
$$

It is clear that $G_{\mathbf{R}}$ is a real form of $G_{\mathbf{C}}$. Since $G_{\mathbf{R}}$ is nilpotent, it has an imaginary spectrum. Therefore $G_{\mathbf{C}}$ admits a right- $G_{\mathbf{R}}$ invariant Kähler form $\omega$ (see [13]). In view of the product structure of $G$ we may assume that $\omega$ restricted to the $w$-direction is the standard Kähler form $d w \wedge d \bar{w}$. Take the 
discrete subgroup $\Gamma$ of $G_{\mathbf{C}}$ generated by $G_{\mathbf{Z}}$ and the element

$$
\left(\begin{array}{cccc}
1 & 0 & \sqrt{2} & i \sqrt{3} \\
0 & 1 & 0 & 0 \\
0 & 0 & 1 & 0 \\
0 & 0 & 0 & 1
\end{array}\right) \in G_{\mathbf{C}}
$$

Then $\omega$ is right $\Gamma$-invariant, i.e., $G_{\mathbf{C}} / \Gamma$ is a Kähler manifold. Let

$$
C=\left\{\left(\begin{array}{cccc}
1 & 0 & z & w \\
0 & 1 & 0 & 0 \\
0 & 0 & 1 & 0 \\
0 & 0 & 0 & 1
\end{array}\right), z, w \in \mathbf{C}\right\} \subset G_{\mathbf{C}}
$$

i.e., $C$ is the center of $G_{\mathbf{C}}$. The base of the fibration $G_{\mathbf{C}} / \Gamma \rightarrow G_{\mathbf{C}} / C \cdot \Gamma$ is $\mathbf{C}^{*} \times \mathbf{C}^{*}$ and the fiber is isomorphic to the Cousin group $\mathbf{C}^{2} / \tilde{\Gamma} ; \tilde{\Gamma}=$ $\left(\left(\begin{array}{l}1 \\ 0\end{array}\right),\left(\begin{array}{l}0 \\ 1\end{array}\right),\left(\begin{array}{c}\sqrt{2} \\ i \sqrt{3}\end{array}\right)\right)_{\mathbf{z}} \subset \mathbf{C}^{2}$. Since $\Gamma$ is not abelian this bundle cannot be (even topologically) trivial.

(b) For this example let

$$
G=\left\{\left(\begin{array}{ccc}
1 & x & z \\
& 1 & y \\
& & 1
\end{array}\right): x, y, z \in \mathbf{C}\right\}
$$

be the complex three-dimensional Heisenberg group. Consider the discret subgroup

$$
\Gamma=\left\{\left(\begin{array}{ccc}
1 & m & k+i l \\
0 & 1 & m \\
0 & 0 & 1
\end{array}\right): n, m, k, l \in \mathbf{Z}\right\}
$$

and let

$$
C=\left\{\left(\begin{array}{ccc}
1 & 0 & z \\
0 & 1 & 0 \\
0 & 0 & 1
\end{array}\right), z \in \mathbf{C}\right\}
$$

i.e., the center and commutator group of $G$. The fibration $G / \Gamma \rightarrow G / C \cdot \Gamma$ realizes $G / \Gamma$ as a torus bundle over $\mathbf{C}^{*} \times \mathbf{C}^{*}$.

The subgroup

$$
G_{0}:=\left\{\left(\begin{array}{ccc}
1 & x & z \\
0 & 1 & y \\
0 & 0 & 1
\end{array}\right): x, y \in \mathbf{R}, z \in \mathbf{C}\right\} \subset G
$$

contains $\Gamma$ cocompact. Thus $\left(\Gamma, G_{0}, G\right)$ is nilpotent $C R S$. Since $m:=\mathfrak{g}_{0} \cap$ $i \mathfrak{g}_{0}=\mathfrak{g}_{0}^{\prime}+i \mathfrak{g}_{0}^{\prime}$, we have $\mathfrak{g}_{0}^{\prime} \cap m \neq(0)$. Hence it follows by Remark 3.4 that $G / \Gamma$ is not Kähler. 


\section{Hypersurfaces in solvmanifolds}

Let $X=G / H$ be a solvmanifold and let

$$
\pi: X=G / H \rightarrow G / I=Z
$$

denote the hypersurface reduction (see $\S 1$ ). Then $Z$ is separable by hypersurfaces and every hypersurface in $X$ is the preimage of one in $Z$, i.e., $\mathcal{H}(X)=\pi^{*} \mathcal{H}(Z)$. Thus in order to describe $\mathcal{H}(X)$ it is sufficient to understand the manifold $Z$.

It turns out that a complex solvmanifold $X$, which is locally separable by global hypersurfaces (see $\S 1$ ), inherits the same structure as a Kählerian solvmanifold.

Theorem 1. Let $X=G / H$ be a complex solvmanifold, which is locally separable by global hypersurfaces. Then the holomorphic reduction $X \stackrel{F}{\rightarrow} Y$ has a Stein base $Y$ and the fiber $F$ is a Cousin group. Moreover $\pi_{1}(X)$ contains a nilpotent subgroup of finite index.

The basic ingredient for the proof of Theorem 1 is

Theorem 2. Let $G$ be an (arbitrary) complex Lie group and $\Gamma \subset G$ a discrete subgroup. If $X=G / \Gamma$ is locally separable by global hypersurfaces, then $X$ admits an exhaustion $\left(U_{m}\right)_{m \in \mathbf{N}}$ and a sequence $\left(\omega_{m}\right)_{m \in \mathbf{N}}$ of real closed positive semidefinite $(1,1)$ forms, such that $\omega_{m} \mid U_{m}$ is positive definite. If $X$ is separable by meromorphic functions, then $X$ is Kähler.

Corollary 3. Let $X=G / \Gamma$ be given as in Theorem 2. Then $X$ is weakly Kählerian.

Before we prove Theorem 2, we show how it implies Theorem 1.

Proof of Theorem 1. The proof is almost exactly the same as that of Theorem 4.1. If $H$ is discrete in $G$ then Theorem 1 immediately follows from Corollary 3 and 4.1. If $H$ is not discrete, let $G_{a}$ denote a regular algebraic hull of $G$ (see $\S 4$ ). Then $G_{a} / H$ is also locally separable by global hypersurfaces. Let $N=N_{G_{a}}\left(H^{0}\right)$. This is an algebraic subgroup of $G_{a}$ (see [12]) and $H / H^{0}$ is a discrete subgroup of $N / H^{0}$. Since $N^{0}$ is also algebraic and since we may assume $H \subset G_{a}(H)$ (see the proof of 4.1), we also may assume that $H \subset N_{0}$. Thus by 4.1 and Corollary 3 we know that the holomorphic reduction $N^{0} / H \rightarrow N^{0} / J$ has a Stein base and a Cousin group as fiber. Since $G_{a} / N^{0}$ is Stein, it follows that $G_{a} / J$ is Stein and $G_{a} / H \rightarrow G_{a} / J$ is the holomorphic reduction. Thus we have $J \subset G, G / J$ is Stein and $G / H \rightarrow G / J$ is the holomorphic reduction. Moreover by 4.1 we have that $H / H^{0}$ contains a nilpotent subgroup of finite index. Thus the same is true for $\pi_{1}(G / H) \cong H / H^{0}$ (for details see $\S 4$; in particular the proof of 4.1 ). q.e.d. 
We now come to the

Proof of Theorem 2. At first we proof the part of the theorem where $X$ is assumed to be locally separable by global hypersurfaces.

Let $p=\Gamma \in G / \Gamma$. By assumption it is possible to construct a hypersurface $H$ such that every irreducible component $H_{i}$ of $H$ is smooth in $p$ and $\bigcap_{i=1}^{n} H_{i}$ contains $p$ as an isolated point $\left(n=\operatorname{dim}_{\mathbf{C}} X\right)$. Let $T_{H}$ be the closed, positive $(1,1)$ current defined by $H$, i.e.,

$$
T_{H}(\varphi):=\int_{H^{*}} \varphi, \quad \varphi \in A_{c}^{n-1, n-1}(X),
$$

where $H^{*}$ is the set of smooth points of $H$ and $A_{c}^{p, q}(X)$ the space of compactly supported $(p, q)$-forms on $X$.

Claim. For every $\eta \in A_{c}^{n-1,0}(X)$ such that $p \in \overbrace{\operatorname{supp}(\eta)}^{\circ}$ we have

$$
\left(\frac{i}{2}\right)^{n-1}(-1)^{(n-1)(n-2) / 2} T_{H}(\eta \wedge \bar{\eta})>0 \text {. }
$$

Proof of the Claim. Take a coordinate patch $\left(U, z=\left(z_{1}, \cdots, z_{n}\right)\right)$ centered at $p$ such that $H_{i} \cap U=\left\{z_{i}=0\right\}$. We may assume that $\operatorname{supp} \eta \subset U$. Let $\eta=\sum_{k} \eta_{k} d z_{1} \wedge \cdots \wedge d \hat{z}_{k} \wedge \cdots \wedge d z_{n}$, where (without loss of generality) $\eta_{1}(p) \neq 0$. (The notation $d z_{1} \wedge \cdots \wedge d \hat{z}_{k} \wedge \cdots \wedge d z_{n}$ means that $d z_{k}$ is omitted.) Then we have

$\eta \wedge \bar{\eta}=\sum_{k=1}^{n} \eta_{k} \bar{\eta}_{k} d z_{1} \wedge \cdots \wedge d \hat{z}_{k} \wedge \cdots \wedge d z_{n} \wedge d \bar{z}_{1} \wedge \cdots \wedge d \hat{\bar{z}}_{k} \wedge \cdots \wedge d \bar{z}_{n}+R$

where $R$ denotes the sum over the mixed components of $\eta \wedge \bar{\eta}$. For such a component $\eta_{k l}=\eta_{k} \bar{\eta}_{l} d z_{1} \wedge \cdots \wedge d \hat{z}_{k} \wedge \cdots \wedge d z_{n} \wedge d \bar{z}_{1} \wedge \cdots \wedge d \hat{\bar{z}}_{l} \wedge \cdots \wedge d \bar{z}_{n}$, $k \neq 1$, it is clear that

$$
\int_{H_{m}=\left\{z_{m}=0\right\}} \eta_{k l}=0 \text { for all } m=1, \cdots, n .
$$

Hence $T_{H}(\eta \wedge \bar{\eta})$ reduces to

$\sum_{k=1}^{n} \int_{H_{k}=\left\{z_{k}=0\right\}}\left|\eta_{k}\right|^{2} d z_{1} \wedge \cdots \wedge d \hat{z}_{k} \wedge \cdots \wedge d z_{n} \wedge d \bar{z}_{1} \wedge \cdots \wedge d \hat{\bar{z}}_{k} \wedge \cdots \wedge d \bar{z}_{n}$ and thus $\eta_{1}(p) \neq 0$ implies the claim.

Since $\Gamma$ is a discrete subgroup of $G$, i.e. the $\pi: G \rightarrow G / \Gamma$ is a covering, we may consider $T_{H}$ as a right $\Gamma$ invariant closed, real, positive $(1,1)$ current on $G$, which is strictly positive on forms $\eta \wedge \bar{\eta}, \eta \in A_{c}^{(n-1,0)}(G)$ such that $\operatorname{supp}(\eta) \cap \Gamma \neq \varnothing$, i.e., for those $\eta$ we have

$$
\left(\frac{i}{2}\right)^{n-1}(-1)^{(n-1)(n-2) / 2} T_{H}(\eta \wedge \bar{\eta})>0 \text {. }
$$


Now let $\left(\mu_{1}, \cdots, \mu_{n}\right)$ denote a basis of the right invariant holomorphic 1-forms on $G$. Then $(i / 2)^{n} \mu_{1} \wedge \bar{\mu}_{1} \wedge \cdots \wedge \mu_{n} \wedge \bar{\mu}_{n}=\mu$ is a right invariant volume on $G$. For $\alpha \in C_{c}^{\infty}(G)$ let

$$
\begin{array}{r}
h_{k l}(\alpha)=\left(\frac{i}{2}\right)^{n-1}(-1)^{n+k+l+1}(-1)^{n(n-1) / 2} T_{H}\left(\alpha \mu_{1} \wedge \cdots \wedge \hat{\mu}_{k} \wedge \cdots \wedge \mu_{n}\right. \\
\left.\wedge \bar{\mu}_{1} \wedge \cdots \wedge \hat{\bar{\mu}}_{1} \wedge \cdots \wedge \bar{\mu}_{n}\right) .
\end{array}
$$

Then we have

$$
T_{H}=\frac{i}{2} \sum_{k, l} h_{k l} \mu_{k} \wedge \bar{\mu}_{l},
$$

and the right $\Gamma$ invariance of $T_{H}$ implies that the distributions $h_{k l}$ are also right $\Gamma$ invariant, i.e., for every $\gamma \in \Gamma$ and every $\alpha \in C_{c}^{\infty}(G)$ one has

$$
r_{\gamma}^{*} h_{k l}(\alpha)=h_{k l}\left(r_{\gamma}^{*} \alpha\right)=h_{k l}(\alpha) \text {. }
$$

Moreover it is clear that $\left(h_{k l}\right)_{k, l}$ is a Hermitian matrix of distributions and that

$$
\sum_{k, l} h_{k l}\left(\alpha_{k} \cdot \bar{\alpha}_{l}\right) \geq 0
$$

for every $\alpha=\left(\alpha_{1}, \cdots, \alpha_{n}\right) \in\left(C_{c}^{\infty}(G)\right)^{n}$. Moreover, by $(*)$ we have $\sum_{k, l} h_{k l}\left(\alpha_{k} \cdot \bar{\alpha}_{l}\right)>0$ if $\left.\overparen{\operatorname{supp}\left(\alpha_{j}\right.}\right) \cap \Gamma \neq \varnothing$ for one $j \in\{1, \cdots, n\}$. In particular it follows that for every real positive function $\alpha \in C_{c}^{\infty}(G)$ the matrix $\left(h_{k l}(\alpha)\right)_{k, l}$ is positive semi-definite and positive definite if $\overbrace{\operatorname{supp}(\alpha)}^{\circ}) e$.

Now we construct a sequence $\omega_{m}$ of right $\Gamma$ invariant $(1,1)$ forms as follows. Take an exhaustion $\left\{K_{m}\right\}_{m \in \mathbf{N}}$ of $G$ with relatively compact subsets $K_{m}$ centered at $e$, i.e. $K_{m} \subset \subset K_{m+1}, \bigcup_{m \in \mathbf{N}}=G$ and $e \in K_{1}$. Further let $\chi_{m}$ denote a subordinate sequence of cut-off functions, i.e., $\left.\chi_{m}\right|_{K_{m}} \equiv 1$ and $\operatorname{supp} \chi_{m} \subset \subset K_{m+1}$.

We take the convolutions

$$
\omega_{m}=\chi_{m} * T_{H}=\int_{G} \chi_{m}(g) l_{g}^{*} T_{H} \mu(g)
$$

The form $\omega_{m}$ is smooth. In order to describe its coefficients with respect to the base $\mu_{k} \wedge \bar{\mu}_{l}$ we need some definitions.

Let $l_{g}$ denote the left multiplication $x \mapsto g \cdot x$ on $G$ and $\tau$ the mapping $x \mapsto x^{-1}$.

For a distribution $h$ and a compactly supported smooth function $\phi$ on $G$ let

$$
\phi * h(g)=h\left(l_{g}^{*}(\phi \circ \tau)\right) \text {. }
$$

Since $\phi$ is compactly supported and smooth, the function $\phi * h$ is likewise smooth. Note that $\phi * h$ is right $\Gamma$ invariant, whenever $h$ is right $\Gamma$ invariant. 
There is a nonsingular matrix of smooth functions $\left(a_{k}^{i}\right)_{1 \leq i, k \leq n}$ on $G$ such that

$$
l_{g}^{*}\left(\mu_{1} \wedge \cdots \wedge \hat{\mu}_{k} \wedge \cdots \wedge \mu_{n}\right)=\sum_{i=1}^{n} a_{k}^{i} \mu_{1} \wedge \cdots \wedge \hat{\mu}_{i} \wedge \cdots \mu_{n} .
$$

An explicit computation of the equation $\chi_{m} * T_{H}(\eta)=\int_{G} \omega_{m} \wedge \eta$ shows that

$$
\omega_{m}=\frac{i}{2} \sum_{k, l=1}^{n} h_{k l}^{m} \mu_{k} \wedge \bar{\mu}_{l}
$$

where

$$
h_{k l}^{m}=(-1)^{k+l} \sum_{i, j=1}^{n}(-1)^{i+j}\left(\chi_{m} a_{k}^{i} \bar{a}_{l}^{j}\right) * h_{i j} .
$$

For $v=\left(v_{1}, \cdots, v_{n}\right) \in \mathbf{C}^{n}$ let

$$
\alpha_{i, g}=\sum_{\nu=1}^{n}(-1)^{\nu+i} l_{g}^{*}\left(\chi_{m}^{1 / 2} a_{\nu}^{i} \circ \tau\right) v_{\nu}
$$

Then we get

$$
\sum_{k, l=1}^{n} h_{k l}^{m}(g) v_{k} \bar{v}_{l}=\sum_{i, j=1}^{n} h_{i j}\left(\alpha_{i, g} \bar{\alpha}_{j, g}\right) .
$$

Just as $\left(a_{\nu}^{i}\right)$ the matrix $\left((-1)^{\nu+i} a_{\nu}^{i}\right)$ is nonsingular everywhere on $G$.

Hence for every $g \in K_{m}$ there is an $i_{0}$, such that

$$
\alpha_{i_{0}, g}(h)=\sum_{\nu=1}^{n}(-1)^{\nu+i_{0}} \chi_{m}^{1 / 2} a_{\nu}^{i_{0}}\left(g h^{-1}\right)
$$

does not vanish in an open neighborhood of $h=e$. This shows that $\left(h_{k l}^{m}(g)\right)$ is positive semidefinite and in particular positive definite for $g \in K_{m}$.

The forms $\omega_{m}$ are right $\Gamma$-invariant and may be considered as a sequence of $(1,1)$-forms on $G / \Gamma$ and $U_{m}=K_{m} \cdot \Gamma$ may be considered as an exhaustion of $G / \Gamma$. The forms $\omega_{m}$ are closed, since they are $C^{\infty}$ and are closed in the sense of currents. For this note that $(\mu(g)$ being the right invariant volume defined above) for $\alpha \in A_{c}^{n-1, n-1}(G)$ we have

$$
\int_{G} \omega_{m} \wedge \alpha=\left(\chi_{m} * T_{H}\right)(\alpha)
$$

where

$$
\chi_{m} * T=\int_{G} \chi_{m}(g)\left(l_{g}^{*} T\right) \mu(g)
$$

Thus the formula

$$
d\left(\chi_{m} * T\right)=\chi_{m} * d T
$$

implies the closedness of $\omega_{m}$ and the first part of the theorem is proved. 
For the second part the separability by meromorphic functions implies the existence of a meromorphic map

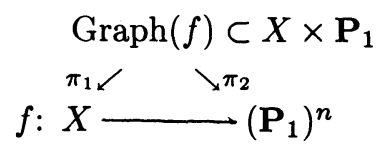

$\left(n=\operatorname{dim}_{\mathbf{C}} X\right)$, which is biholomorphic in the complement of a proper analytic subset of $X$.

Thus a Kähler form $\omega$ on $\left(\mathbf{P}_{1}\right)^{n}$ defines a closed, positive $(1,1)$-current $T$ on $X$ in the following way: For $\varphi \in A_{c}^{n-1, n-1}(X)$ let

$$
T(\varphi):=\int_{\operatorname{Graph}(f)^{*}} \pi_{1}^{*} \varphi \wedge \pi_{2}^{*} \omega,
$$

where $\operatorname{Graph}(f)^{*}$ denotes the set of smooth points of $\operatorname{Graph}(f)$. The current $T$ is strictly positive on $G$ in the sense that for every $\eta \in A_{c}^{n-1,0}(X)$ we have

$$
\left(\frac{i}{2}\right)^{n-1}(-1)^{(n-1)(n-2) / 2} T(\eta \wedge \bar{\eta})>0 \text {. }
$$

This is clear since $\omega$ is a Kähler form $\left(\mathbf{P}_{1}\right)^{n}$ and $f$ is biholomorphic in the complement of a proper analytic set. Let $0 \neq \varphi \in C_{c}^{\infty}(G)$ be a positive, compactly supported function and write

$$
T=\frac{i}{2} \sum_{k, l=1}^{n} h_{k l} \mu_{k} \wedge \bar{\mu}_{l} .
$$

Following the first part of the proof define the convolutions $\varphi * h_{k l}=\tilde{h}_{k l}$ and the associated closed $(1,1)$-form $\tilde{\omega}=\tilde{\omega}=\varphi * T$. The form $\omega$ is right $\Gamma$ invariant and strictly positive by using the same arguments as above. Thus $\tilde{\omega}$ is a Kähler form on $X$ and the theorem is proved.

Remark 4. We do not know yet whether the assumption (concerning hypersurfaces) in Theorem 2 implies that $X$ is Kähler. For instance one could consider $f \cdot \chi_{n}$ instead of $\chi_{n}$ in the proof of Theorem 2, where $f$ denotes a strictly positive function integrable with respect to the measure $\mu$. The question is whether $f$ can be chosen in such a way that $\lim _{m \rightarrow \infty} \omega_{m}$ exists. We also do not know whether the assumption of discrete isotropy in Theorem 2 can be dropped.

\section{On the conjecture of Ahiezer}

In this section we prove the conjecture of Ahiezer (see $§ 1$ ) for the case where $X=G / H$ is a solvmanifold. 
Theorem 1. Let $G$ be a connected solvable complex Lie group and $H \subset G$ an arbitrary subgroup. Suppose $\mathcal{O}(G)^{H}=$ C. Then $\mathcal{H}(G)^{H}=\mathcal{H}(G)^{G^{\prime} H}$.

Proof. We may assume that $G$ is simply connected. Let $\bar{H}$ be the smallest closed complex subgroup of $G$ containing $H$. By a result of [19] it follows that $\mathcal{H}(G)^{H}=\mathcal{H}(G)^{\bar{H}}$ and $\mathcal{O}(G)^{\bar{H}}=$ C. Thus we have $\mathcal{H}(G)^{H}=\alpha^{*} H(G / \bar{H})$, where $\alpha: G \rightarrow G / \bar{H}$ denotes the quotient map defined by $\bar{H}$. Let $\pi: G / \bar{H} \rightarrow$ $G / I$ denote the hypersurface reduction of $G / \bar{H}$. Then we have $O(G / I)=\mathbf{C}$ and $\mathcal{H}(G / \bar{H})=\pi^{*} \mathcal{H}(G / I)$ (see $\S 1$ ). Since $O(G / I)=\mathrm{C}$, it follows that $I^{0} \triangleleft G$ and the simply connected solvable complex Lie group $\dot{G} / I^{0}$ acts almost freely on $G / I$. Thus Theorem 5.1 implies $G^{\prime} \subset I^{0}$ and hence the theorem.

Corollary 2. Let $X=G / H$ be a solvmanifold. Suppose $O(X)=\mathbf{C}$. Then the base $Y=G / J$ of the hypersurface reduction of $X$ is a Cousin group.

Remark. A Cousin group, which is separable by hypersurfaces, is already meromorphically separable (see [18, Anhang]). Therefore the meromorphic and the hypersurface reduction of $X$ (as in Corollary 2) coincide.

\section{References}

[1] D. Ahiezer, Invariant analytic hypersurfaces in complex nilpotent Lie groups, Ann. Global Anal. Geom. 2 (1984) 129-140.

[2] W. Barth \& M. Otte, Über fast uniforme Untergruppen komplexer Liegruppen und auflösbare komplexe Mannigfaltigkeiten, Comment. Math. Helv. 44 (1969) 269-281.

[3] A. Borel, Linear algebraic groups, Benjamin, New York, 1969.

[4] A. Borel \& R. Remmert, Über kompakte homogene Kählersche Mannigfaltigkeiten, Math. Ann. 145 (1962) 429-439.

[5] P. Cousin, Sur les fonctions triplement périodiques de deux variables, Acta Math. 10 (1910) 105-232.

[6] H. Grauert, Analytische Faserungen über holomorph vollständigen Räumen, Math. Ann. 135 (1958) 263-273.

[7] H. Grauert \& R. Remmert, Über kompakte homogene komplexe Mannigfaltigkeiten, Arch. Math. 13 (1962) 498-507.

[8] B. Gilligan \& A. T. Huckleberry, On non-compact complex nilmanifolds, Math. Ann. 238 (1978) 39-49.

[9] G. Hochschild \& G. D. Mostow, On the algebra of representative functions of an analytic group. II, Amer. J. Math. 86 (1964) 869-887.

[10] A. T. Huckleberry \& G. A. Margulis, Invariant analytic hypersurfaces, Invent. Math. 71 (1983) 235-240.

[11] A. T. Huckleberry \& E. Oeljeklaus, Classification theorems for almost homogeneous spaces, Revue de l'Institut Elie Cartan 9 (Nancy), Janvier 1984.

[12] __ On holomorphically separable complex solv-manifolds, Ann. Inst. Fourier (Grenoble) 36 (1986) 57-65.

[13] J. J. Loeb, Actions d'une forme de Lie reele d'un groupe de Lie complexe sur les fonctions plurisousharmoniques, Ann. Inst. Fourier (Grenoble) 35 (1985) 59-97.

[14] J. J. Loeb, K. Oeljeklaus \& W. Richthofer, A decomposition theorem for complex nilmanifolds, Canad. Math. Bull. 30 (1987).

[15] Y. Matsushima, On discrete subgroups and homogeneous spaces of nilpotent groups, Nagoya Math. J. 2 (1951) 95-110. 
[16] Y. Matsushima \& A. Morimoto, Sur certains espaces fibrés holomorphes sur une variété de Stein, Bull. Soc. Math. France 88 (1960) 137-155.

[17] G. D. Mostov, Factor spaces of solvable groups, Ann. of Math. 60 (1954) 1-27.

[18] K. Oeljeklaus, Hyperflächen und Geradenbündel auf homogenen komplexen Mannigfaltigkeiten, Dissertation, Bochum 1984, Schriftenreihe Math. Inst. Univ. Münster, Ser. 2, Bd. 36, 1985.

[19] W. Richthofer, Homogene CR-Mannigfaltigkeiten, Dissertation, Bochum, 1985.

[20] R. Remmert \& T. van de Ven, Zur Funktionentheorie homogener komplexer Mannigfaltigkeiten, Topology 2 (1963) 137-157.

[21] J. Snow, Complex solvmanifolds of dimension two and three, Thesis, Notre Dame University, 1979.

[22] D. Snow, Stein quotients of complex Lie groups, Manuscripta Math. 50 (1985) 185-214.

[23] H. C. Wang, Complex parallelisable manifolds, Proc. Amer. Math. Soc. 5 (1954) 771, 776.

\section{RUHR-UNIVERSITÄT BOCHUM, WEST GERMANY}


